\title{
JOINED DISSIMILAR ORTHOTROPIC ELASTIC CYLINDRICAL MEMBRANES UNDER INTERNAL PRESSURE AND LONGITUDINAL TENSION
}

\author{
V. G. HART and JINGYU SHI ${ }^{1}$
}

(Received 12 January 1991; revised 30 July 1991)

\begin{abstract}
Following work in an earlier paper, the theory of finite deformation of elastic membranes is applied to the problem of two initially-circular semi-infinite cylindrical membranes of the same radius but of different material, joined longitudinally at a cross-section. The body is inflated by constant interior pressure and is also extended longitudinally. The exact solution found for an arbitrary material is now specialised to the orthotropic case, and the results are interpreted for forms of the strain-energy function introduced by Vaishnav and by How and Clarke in connection with the study of arteries. Also considered in this context is the similar problem where two semi-infinite cylindrical membranes of the same material are separated by a cuff of different material. Numerical solutions are obtained for various pressures and longitudinal extensions. It is shown that discontinuities in the circumferential stress at the joint can be reduced by suitable choice of certain coefficients in the expression defining the strain-energy function. The results obtained here thus solve the problem of static internal pressure loading in extended dissimilar thin orthotropic tubes, and may also be useful in the preliminary study of surgical implants in arteries.
\end{abstract}

\section{Introduction}

Since the theory of large elastic deformations of membranes was formulated by Adkins and Rivlin [1], who also gave solutions to some problems in which the deformation was axially symmetric, it has been applied to the solution of additional problems of this type. Kydoniefs and Spencer [7] gave the exact solution to the differential equations governing the axisymmetric deformations of a cylindrical Mooney membrane. Pipkin [9] gave the integration

\footnotetext{
${ }^{1}$ Department of Mathematics, University of Queensland, Australia.

(C) Copyright Australian Mathematical Society 1992, Serial-fee code 0334-2700/92
} 
of one of the equilibrium equations governing axisymmetric deformation of a cylindrical membrane composed of general homogeneous incompressible elastic material. Subsequently, Kydoniefs [6] derived exact solutions to two mixed boundary-value problems, in which a cylindrical membrane encloses a rigid body and is axisymmetrically deformed by internal pressure and axial force.

In the authors' previous paper [3], using Pipkin's [9] results, the axisymmetric deformations were studied of two initially-circular cylindrical membranes, composed of different isotropic elastic materials joined together at a cross section and extended longitudinally under constant internal pressure. The solutions are exact in the sense that the equilibrium equations are integrated analytically, numerical methods being used only for the determination of one of the variables describing the deformed configuration. The exact solutions are valid for general homogeneous isotropic incompressible elastic materials and the examples were calculated for Mooney material.

In this paper, we formulate these problems for general homogeneous elastic material rather than for a particular class (isotropic) of materials. Both isotropic and orthotropic materials are involved in our examples. While the solutions found here are valid for the mechanical problem posed of dissimilar elastic cylinders of any orthotropic type, under tension and constant internal pressure, we have chosen the material constants in our examples from previous experimental work on natural arteries and artificial grafts. Thus the results found can be regarded also as supplying preliminary information on the subject of an implant of an artificial graft into an artery-albeit under static rather than dynamic loading conditions.

In Section 2, we summarise first the field equations governing the deformation of an initially circular cylindrical membrane of homogeneous incompressible elastic material, subjected to an axial tensile force and internal pressure. Then we outline the scheme of solving a specific boundary-value problem. These equations and the proposed scheme are applicable to general materials.

In Section 3, we set up the conditions when two cylindrical membranes of different elastic materials are joined together. As examples, in Section 4 we discuss the situation in which two semi-infinite membranes are joined together. In Section 5, we consider the link of two semi-infinite membranes with the same material by a cuff composed of different material.

\section{Axisymmetrical deformations of initially-cylindrical membranes}

Axisymmetrical deformations of initially-cylindrical membranes are considered. The membranes are composed of homogeneous incompressible elas- 

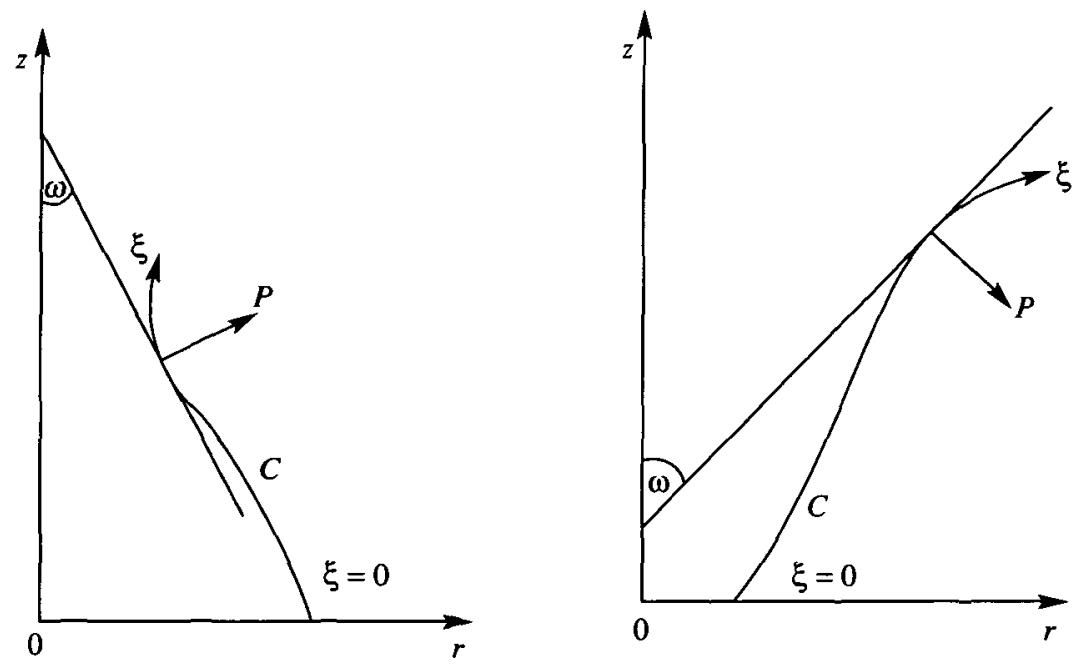

FIGURE 1. Axial sections of the deformed membrane (with $z_{0}=0$ ).

tic material and have uniform thickness $2 h_{0}$. The authors' notation in their previous paper will be used in the following.

We assume that the deformation is caused by internal pressure $P_{i}$, measured per unit area of the deformed membrane, and axial force $F_{e}$ at the ends. The pressure $P_{i}$ may vary longitudinally, but not circumferentially so that it can produce an axisymmetrical deformation. The deformations are referred to cylindrical polar coordinates; $(\rho, \Theta, \eta), \rho=$ constant, are the coordinates of a particle in the undeformed configuration which has coordinates $(r, \theta, z)$, after the deformation, given by

$$
r=r(\eta), \quad \theta=\theta, \quad z=z(\eta) .
$$

Therefore the deformed membrane could be generated by rotating a continuous curve $C$ about the $z$-axis. It is assumed that the tangent to $C$ is nowhere parallel or perpendicular to the $z$-axis, except perhaps at end points of $C$; otherwise $C$ may be divided into segments for which this condition does hold. The deformed configuration is, therefore, one of those illustrated in Figure 1. The arc length of $C$ is denoted by $\xi$ and the angle between the tangent to $C$ and the $z$-axis by $\omega$, where $0 \leq \omega \leq \pi / 2$.

Since the deformation is axially symmetric, the principal directions of strain coincide with the meridian, the lines of latitude and the normal to the deformed surface. The principal extension ratios corresponding to these directions are denoted by $\lambda_{1}, \lambda_{2}, \lambda_{3}$, respectively, and are given by

$$
\lambda_{1}=d \xi / d \eta, \quad \lambda_{2}=r / \rho, \quad \lambda_{3}=\left(\lambda_{1} \lambda_{2}\right)^{-1}=h / h_{0},
$$


where $2 h$ is the variable thickness of the deformed membrane and the incompressibility condition $\lambda_{1} \lambda_{2} \lambda_{3}=1$ has been used. The general strain-energy function for an incompressible elastic membrane can be expressed by

$$
W=W\left(\lambda_{1}, \lambda_{2}\right) \text {. }
$$

If $T_{1}$ and $T_{2}$ denote the stress resultants in the deformed membrane, in the directions of the tangents to the meridian curves and the curves of latitude respectively, then we have

$$
\begin{aligned}
& T_{1}=2 h \lambda_{1} \frac{\partial W}{\partial \lambda_{1}}=2 h_{0} \lambda_{2}^{-1} \frac{\partial W}{\partial \lambda_{1}}, \\
& T_{2}=2 h \lambda_{2} \frac{\partial W}{\partial \lambda_{2}}=2 h_{0} \lambda_{1}^{-1} \frac{\partial W}{\partial \lambda_{2}} .
\end{aligned}
$$

Two quantities which must be used to study deformations of membrane are the principal curvatures, $\kappa_{1}$ and $\kappa_{2}$, of the surface of the membrane, $\kappa_{1}$ being the curvature of the meridian curve. A principal curvature is positive when the corresponding centre of the curvature is on the same side of the surface as the inward pointing normal. Then

$$
\kappa_{1}=d(\cos \omega) / d r, \quad \kappa_{2}=\cos \omega / r .
$$

The equations of equilibrium can be written in the form

$$
d\left(r T_{1}\right) / d r=T_{2}, \quad \kappa_{1} T_{1}+\kappa_{2} T_{2}=P_{i} .
$$

The equations (2.1)-(2.6) govern the axisymmetrical deformation of an initially cylindrical membrane of homogeneous incompressible elastic material. The angle $\omega$ and the arc length $\xi$ can be expressed in terms of $r$ and $z$. Then the equations in (2.6) are the coupled ordinary differential equations for $r$ and $z$ if $\eta$ is chosen as the independent variable. Alternatively, we can choose $\lambda_{2}$, or $r\left(=\rho \lambda_{2}\right)$ from $(2.2)_{2}$, as the independent variable, and the equations in (2.6) may be used to determine the extension ratio $\lambda_{1}$ through (2.4) and the angle $\omega$. Then from (2.2) and the geometry in Figure 1 we can determine $z, \xi$ and $\eta$. The latter procedure will be followed in this paper.

Substituting (2.4) into the first of (2.6) and making use of (2.2), we have

$$
\frac{d}{d \lambda_{2}}\left(\frac{\partial W}{\partial \lambda_{1}}\right)=\lambda_{1}^{-1} \frac{\partial W}{\partial \lambda_{2}} \text {. }
$$

It has been shown that when the initial shape is a circular cylinder (see Pipkin [9]), this can be integrated to give

$$
W-\lambda_{1} \frac{\partial W}{\partial \lambda_{1}}=A
$$


where $A$ is an integration constant. From the last equation, $\lambda_{1}$ can be determined in terms of $\lambda_{2}$ and the constant $A$ when the strain-energy function $W\left(\lambda_{1}, \lambda_{2}\right)$ is specified. Substituting (2.4), (2.5) into the second of (2.6) and using (2.2) and the first of (2.6) gives rise to

$$
\frac{d}{d \lambda_{2}}\left(\frac{\partial W}{\partial \lambda_{1}} \cos \omega\right)=\rho P_{i} \lambda_{2}\left(2 h_{0}\right)^{-1}
$$

which determines $\omega$ as follows:

$$
\cos \omega=\left\{\rho \int P_{i} \lambda_{2} d \lambda_{2}\right\} /\left\{2 h_{0} \frac{\partial W}{\partial \lambda_{1}}\right\}
$$

If the pressure is the same everywhere, $P_{i}=P_{0}$ (constant), the case in which we are interested in this paper, then this becomes

$$
\cos \omega=\left\{\rho P_{0} \lambda_{2}^{2} / 2+B\right\} /\left\{2 h_{0} \frac{\partial W}{\partial \lambda_{1}}\right\}
$$

where $B$ is another integration constant.

The constants $A$ and $B$ can be determined in terms of the extension ratios at one of the end sections when both strain-energy function and the external loading are specified. For example, if the extension ratios and the resultant of the axial force at the top end section are $\Lambda_{1 T}, \Lambda_{2 T}$ and $F_{T}$, then, since (2.7) holds along the meridian,

$$
A=\left.\left(W-\lambda_{1} \frac{\partial W}{\partial \lambda_{1}}\right)\right|_{\left(\Lambda_{1 T}, \Lambda_{2 T}\right)}
$$

The balance of the axial components of the loading applied on the part of the membrane between the top end section and any arbitrary section with circumferential extension ratio $\lambda_{2}$ yields

$$
2 \pi \rho \lambda_{2} T_{1} \cos \omega=F_{T}-\pi \rho^{2} P_{0}\left(\Lambda_{2 T}^{2}-\lambda_{2}^{2}\right),
$$

or

$$
\cos \omega=\left\{F_{T} /(\pi \rho)-\rho P_{0}\left(\Lambda_{2 T}^{2}-\lambda_{2}^{2}\right)\right\} /\left\{4 h_{0} \frac{\partial W}{\partial \lambda_{1}}\right\} .
$$

The expression (2.9) also holds at any section. Then, equating (2.9) with (2.11), we have

$$
B=F_{T} /(2 \pi \rho)-\left(\rho P_{0} \Lambda_{2 T}^{2}\right) / 2,
$$

and

$$
\cos \omega=\left\{\pi \rho^{2} P_{0}\left(\lambda_{2}^{2}-\Lambda_{2 T}^{2}\right)+F_{T}\right\} /\left\{4 \pi \rho h_{0}\left(\partial W / \partial \lambda_{1}\right)\right\}
$$

Therefore, (2.7) and (2.12) determine the extension ratio $\lambda_{1}$ and the angle $\omega$ in terms of $\lambda_{2}, \Lambda_{1 T}$ and $\Lambda_{2 T}$. 
From the expression (2.12), at the top end section, we have a relation between the angle $\omega=\Omega_{T}$ and the extension ratios $\Lambda_{1 T}$ and $\Lambda_{2 T}$

$$
\cos \Omega_{T}=F_{T} /\left.\left\{4 \pi \rho h_{0}\left(\partial W / \partial \lambda_{1}\right)\right\}\right|_{\left(\Lambda_{1 T}, \Lambda_{2 T}\right)} .
$$

Thus we can specify any two of the three quantities $\Omega_{T}, \Lambda_{1 T}$ and $\Lambda_{2 T}$ to complete the determination of $\lambda_{1}, \omega$ and the tensions $T_{1}$ and $T_{2}$ through (2.4) in terms of $\lambda_{2}$.

After $\lambda_{1}$ and $\omega$ are determined, the deformed and undeformed configurations can be found. From Figure 1 and (2.2), we have

$$
\begin{aligned}
\left(z-z_{0}\right) / \rho & = \pm \int_{\Lambda_{2}}^{\lambda_{2}} \cot \omega d \lambda_{2}, \\
\xi / \rho & = \pm \int_{\Lambda_{2}}^{\lambda_{2}} d \lambda_{2} / \sin \omega, \\
\eta / \rho & = \pm \int_{\Lambda_{2}}^{\lambda_{2}} d \lambda_{2} /\left(\lambda_{1} \sin \omega\right) .
\end{aligned}
$$

In (2.13) the positive or negative sign is dependent upon whether $\lambda_{2}$ is an increasing or decreasing function of $\xi$ and it has been assumed that $\lambda_{2}=\Lambda_{2}$ and $\xi=\eta=0$ at some section $z=z_{0}$ (in Figure 1 we choose $z_{0}=0$ ). The quantity $\Lambda_{2}$ could be chosen as $\Lambda_{2 T}$, but we prefer to use it as another parameter.

If the total original length between the top end section and the section $z=z_{0}$ is given as $L$, then the third of (2.13) gives a relation between $\Lambda_{1 T}$, $\Lambda_{2 T}$ and $\Lambda_{2}$ :

$$
L / \rho= \pm \int-\Lambda_{2}{ }^{\Lambda_{2 T}} d \lambda_{2} /\left(\lambda_{1} \sin \omega\right)
$$

So if $\Lambda_{2}$ is given by some independent condition, then only one of three quantities $\Lambda_{1 T}, \Lambda_{2 T}$ and $\Omega_{T}$ at the top end section is independent. One point should be mentioned here. According to the assumption made on the curve $C$ at the beginning of this section, $\lambda_{2}$ is a monotonic function of $\xi$. If the section $z=z_{0}$ is not the top end i.e. $L \neq 0$, then $\Lambda_{2 T} \neq \Lambda_{2}$. But if it is found from the independent condition that $\Lambda_{2 T}=\Lambda_{2}$ in magnitude (i.e. the radii are equal), then (2.14) would give the unreasonable result $L=0$, which implies that the integrand in (2.14) is singular at least at one interior section. Since $\lambda_{1}$ is finite, the only possibility for the integrand to be singular is that the angle $\omega$ is zero at one or more sections. Thus we have to divide this part into at least two sub-parts and then study each of them. To find the original length of the whole part, the integral in (2.14) must be broken into at least two parts, each with a singularity at the end of the interval. The approximate method to carry out these singular integrals is the same as that 
which will be used in Section 5. We use the condition $\omega=0$ to determine the circumferential extension ratio at the singular section.

The balance of the axial components of the forces applied on the membrane between the top end section and the section $z=z_{0}$ yields the expression for the axial force acting at the section $z=z_{0}$

$$
F_{z_{0}}=F_{T}-\pi \rho^{2} P_{0}\left(\Lambda_{2 T}^{2}-\Lambda_{2}^{2}\right) \text {. }
$$

Since $\Lambda_{2 T} \neq \Lambda_{2}, F_{z_{0}} \neq F_{T}$.

\section{Joining of two membranes of different materials}

The formulae derived in the preceding section hold in one material. Now we consider the join of two initially circular cylindrical membranes composed of different homogeneous incompressible elastic materials. We assume that the membranes are joined together at section $z=z_{0}$. We subscript $T$ and $B$ to the quantities in the top part $\left(z \geq z_{0}\right)$ and the bottom part $\left(z \leq z_{0}\right)$, respectively, of the joined membrane to simplify the explanation.

At the joining section $z=z_{0}$, we have the continuity conditions

$$
\begin{gathered}
\lambda_{2 T}=\lambda_{2 B}=\Lambda_{2 J}, \\
\omega_{T}=\omega_{B}=\Omega_{J} .
\end{gathered}
$$

The condition (3.1) results from the requirement that the membranes should have the same radii at the joining section $z=z_{0}$, and (3.2) signifies continuity of the slope of the meridian curve, as required by the membrane theory. Then (3.2) implies the continuity of the longitudinal tension: $T_{1 T}=T_{1 B}$, and, in turn, we deduce that

$$
\left.\left(\frac{\partial W_{T}}{\partial \lambda_{1 T}}\right)\right|_{\left(\Lambda_{1 T J}, \Lambda_{2 J}\right)}=\left.\left(\frac{\partial W_{B}}{\partial \lambda_{1 B}}\right)\right|_{\left(\Lambda_{1 B J}, \Lambda_{2 J}\right)},
$$

where $W_{T}=W_{T}\left(\lambda_{1 T}, \lambda_{2 T}\right)$ and $W_{B}=W_{B}\left(\lambda_{1 B}, \lambda_{2 B}\right)$ are strain-energy functions for the top and bottom parts respectively. In (3.3), $\Lambda_{1 T J}$ and $\Lambda_{1 B J}$ are the values of $\lambda_{1 T}$ and $\lambda_{1 B}$ at the joining section, and can be determined from

$$
\begin{aligned}
& \left.\left(W_{T}-\lambda_{1 T} \frac{\partial W_{T}}{\partial \lambda_{1 T}}\right)\right|_{\left(\Lambda_{1 T J}, \Lambda_{2 J}\right)}=\left.\left(W_{T}-\lambda_{1 T} \frac{\partial W_{T}}{\partial \lambda_{1 T}}\right)\right|_{\left(\Lambda_{1 T}, \Lambda_{2 T}\right)}, \\
& \left.\left(W_{B}-\lambda_{1 B} \frac{\partial W_{B}}{\partial \lambda_{1 B}}\right)\right|_{\left(\Lambda_{1 B J}, \Lambda_{2 J}\right)}=\left.\left(W_{B}-\lambda_{1 B} \frac{\partial W_{B}}{\partial \lambda_{1 B}}\right)\right|_{\left(\Lambda_{1 B}, \Lambda_{2 B}\right)},
\end{aligned}
$$

in terms of the extension ratios $\Lambda_{2 J}$ at the joining section, $\Lambda_{1 T}, \Lambda_{2 T}$ at the top end section of the top part and $\Lambda_{1 B}, \Lambda_{2 B}$ at the bottom end section of the bottom part. 
Balancing the axial components of the loading acting on the whole joined membrane, we have the relation between the axial forces $F_{T}$ at the top end section of the top part and $F_{B}$ at the bottom end section of the bottom part

$$
F_{B}=F_{T}-\pi \rho^{2} P_{0}\left(\Lambda_{2 T}^{2}-\Lambda_{2 B}^{2}\right) \text {. }
$$

With (3.3) and (3.6), it can easily be proved that the axial component of the longitudinal tension $T_{1} \cos \omega$ continuously crosses the joining section. But the longitudinal extension ratio $\lambda_{1}$ and the circumferential tension resultant $T_{2}$ may be discontinuous at the joining section.

The equation (3.3) determines $\Lambda_{2 J}$ at the joint. Then we can specify any two of $\Lambda_{1 T}, \Lambda_{2 T}$ and $\Omega_{T}$ at the top end section of the top part and any two of $\Lambda_{1 B}, \Lambda_{2 B}$ and $\Omega_{B}$ at the bottom end section of the bottom part if the original lengths of the two parts are not given (one may be asked to determine the lengths). If we are supplied with the lengths then the number of the independent variables is reduced by one at each end section. Thus we need more conditions to determine the reduced variables. If the lengths are finite, then the conditions are of the form of (2.14). If the lengths are infinite, then we will have other conditions. In the following sections we will discuss various situations in which the lengths are given.

After the extension ratios at the joining and end sections are determined, we can determine the longitudinal extension ratio $\lambda_{1}$, the tension resultants $T_{1}$ and $T_{2}$, and the angle $\omega$ in terms of the circumferential extension ratio $\lambda_{2}$ in the two different parts, and then carry out the integrations in (2.13) with the corresponding parameters to give the deformation.

\section{Join of two semi-infinite cylindrical elastic membranes}

As an example, we discuss in this section the situation in which both membranes are semi-infinite. We assume that the joining section is located at $z=z_{0}=0$. Then $\Lambda_{1 T}, \Lambda_{2 T}, \Lambda_{1 B}$ and $\Lambda_{2 B}$ are the values of the extension ratios as $z$ tends to positive or negative infinity respectively. We expect the join to have no effect on the deformation at large $|z|$. Consequently, at a large distance from the joining section, the membranes will be deformed under the action of the axial force and constant internal pressure and the deformed membranes tend to the circularly cylindrical, i.e. $\Omega_{T}=\Omega_{B}=0$. So $F_{T}$ and $P_{0}$ are balanced with $T_{1 T}$ and $T_{2 T}$ at $z \rightarrow \infty$ respectively, $F_{B}$ and $P_{0}$ with $T_{1 B}$ and $T_{2 B}$ at $z \rightarrow-\infty$ respectively, where $F_{B}$ should satisfy (3.6). 
It follows from the above observation that for $z \rightarrow \infty$ we have

$$
2 \pi \rho \Lambda_{2 T} T_{1 T}=F_{T}, \quad T_{2 T}=P_{0} \rho \Lambda_{2 T} .
$$

Expressing the tension resultants in terms of the strain-energy function $W_{T}$ as in (2.4), we obtain

$$
\frac{\partial W_{T}}{\partial \lambda_{1 T}}=F, \quad \frac{\partial W_{T}}{\partial \lambda_{2 T}}=P \Lambda_{1 T} \Lambda_{2 T},
$$

in which we have put

$$
F=F_{T} /\left(4 \pi \rho h_{0}\right), \quad P=\rho P_{0} /\left(2 h_{0}\right) .
$$

Similarly, at the other end where $z \rightarrow-\infty$, we have, by (3.6)

$$
\frac{\partial W_{B}}{\partial \lambda_{1 B}}=F-P\left(\Lambda_{2 T}^{2}-\Lambda_{2 B}^{2}\right) / 2, \quad \frac{\partial W_{B}}{\partial \lambda_{2 B}}=P \Lambda_{1 B} \Lambda_{2 B} .
$$

After $\Lambda_{1 T}$ and $\Lambda_{2 T}$ are either determined from (4.2) for given $F$ and $P$, or specified (one or both of them) to determine the external loading from (4.2), $\Lambda_{1 B}$ and $\Lambda_{2 B}$ can be found from (4.4). Then (3.3)-(3.5) are the governing equations for the extension ratios $\Lambda_{1 T J}, \Lambda_{2 J}$ and $\Lambda_{1 B J}$ at the joining section. In this situation, the equations for $\Lambda_{1 B}$ and $\Lambda_{2 B}$ are separated from those for $\Lambda_{1 T J}, \Lambda_{2 J}$ and $\Lambda_{1 B J}$.

So far the strain-energy functions take the general form (2.3). To find the numerical values of the extension ratios as well as the tension resultants, we need specific forms of these functions. As indicated in the Introduction, we are interested in the implant of an artificial graft into a natural artery. If both the natural and artificial arteries are sufficiently long that (4.2) and (4.4) hold with very good accuracy, then they can be treated as semi-infinite membranes. Therefore we will treat the top part as natural artery and use the strain-energy function given and tested experimentally by Vaishnav [11]

$$
W_{T}=C_{1} a_{T}^{2}+C_{2} a_{T} b_{T}+C_{3} b_{T}^{2}+C_{4} a_{T}^{3}+C_{5} a_{T}^{2} b_{T}+C_{6} a_{T} b_{T}^{2}+C_{7} b_{T}^{3},
$$

where

$$
a_{T}=\left(\lambda_{2 T}^{2}-1\right) / 2, b_{T}=\left(\lambda_{1 T}^{2}-1\right) / 2
$$

are Green-St. Venant strains in the circumferential and longitudinal directions and the material coefficients are shown on the first row of Table 1.

TABLE 1. The values of the material coefficients of Vaishnav and How-Clarke membranes in the expressions (4.5) and (4.7).

\begin{tabular}{|c|ccccrrr|}
\hline$i$ & 1 & 2 & 3 & 4 & \multicolumn{1}{c}{5} & \multicolumn{1}{c}{6} & \multicolumn{1}{c|}{7} \\
\hline$C_{i}\left(10^{5} \mathrm{~N} / \mathrm{m}^{2}\right)$ & 0.3230 & 0.0340 & 0.2470 & 0.0250 & 0.0680 & 0.8620 & -0.0410 \\
$D_{i}\left(10^{5} \mathrm{~N} / \mathrm{m}^{2}\right)$ & 3.0231 & 2.9227 & 4.1104 & -1.6959 & -1.8700 & -2.8331 & -1.9322 \\
\hline
\end{tabular}


The bottom part is an artificial implant. We first employ an orthotropic graft used in surgery, the experiments on whose material properties were carried out by How and Clarke [4]. The strain-energy function is of the same form as $(4.5)$, i.e.

$$
W_{B}=D_{1} a_{B}^{2}+D_{2} a_{B} b_{B}+D_{3} b_{B}^{2}+D_{4} a_{B}^{3}+D_{5} a_{B}^{2} b_{B}+D_{6} a_{B} b_{B}^{2}+D_{7} b_{B}^{3},
$$

where

$$
a_{B}=\left(\lambda_{2 B}^{2}-1\right) / 2, \quad b_{B}=\left(\lambda_{1 B}^{2}-1\right) / 2
$$

are again Green-St. Venant strains in the circumferential and longitudinal directions and the material coefficients are shown on the second row of Table 1.

As previously mentioned, the resultant tension $T_{2}$ in the circumferential direction may have a discontinuity or jump at the joining section. This jump does exist in the situation when two isotropic cylindrical membranes are joined together (see Hart and Shi [3]). From the results found here the jump also occurs when a How-Clarke artificial graft is joined to a natural artery with the strain-energy function of the form (4.5). Now the question can be asked, how large is the jump if the artificial graft is composed of isotropic elastic material, such as a rubber-like material? So we then use, instead of (4.7), the strain-energy function describing a Mooney material:

$$
W_{B}=C_{1 B}\left(\lambda_{1 B}^{2}+\lambda_{2 B}^{2}+\lambda_{1 B}^{-2} \lambda_{2 B}^{-2}-3\right)+C_{2 B}\left(\lambda_{1 B}^{2} \lambda_{2 B}^{2}+\lambda_{1 B}^{-2}+\lambda_{2 B}^{-2}-3\right)
$$

and carry out some calculations. The material constants we used are $C_{1 B}=$ $2.0 \times 10^{5} \mathrm{~N} / \mathrm{m}^{2}$ and $C_{2 B}=0.4 \times 10^{5} \mathrm{~N} / \mathrm{m}^{2}$. These values were used by Kydoniefs [6] and are close to those measured for a certain type of vulcanised rubber by Rivlin and Saunders [10]. It is found that the jump for the isotropic graft is larger than that for a How-Clarke orthotropic graft under the same conditions at the top end section of the natural artery.

From the mechanical point of view, a large jump in the resultant tension at the joint may be one of the factors which cause failure in surgery. Therefore it is highly desirable to manufacture a graft which can produce a smaller jump at the joining section. So we vary the coefficients in (4.7) and seek those which give smaller jumps than the How-Clarke material. The following examples are constructed with a number of different kinds of material for the bottom part, namely Mooney and How-Clarke materials with varied coefficients.

For the equations in (4.2) with the strain-energy function given in (4.5), we can assume that $F$ and $P$ are known, then we can solve for $\Lambda_{1 T}$ and $\Lambda_{2 T}$. But we prefer to specify $\Lambda_{1 T}$ and $P_{0}$, then solve for $\Lambda_{2 T}$ and the axial force $F$. The reason for this is that there are some observations on the contraction of arteries which were excised from animals as well as human bodies (see Bergel [2] and Pedley [8]). The contraction varies from $15 \%$ to $42 \%$. So we 
specify $\Lambda_{1 T}=1.4$. The internal pressure is specified as $P_{0}=0.10,0.15$ and $0.20\left(\times 10^{5} \mathrm{~N} / \mathrm{m}^{2}\right)$ which are in the range where the experiments were carried out. These values of pressure are also in the physiological range. The original thickness and radii of the cylindrical membranes are given as $2 h_{0}=5.0 \times 10^{-4} \mathrm{~m}$ and $\rho=2.5 \times 10^{-3} \mathrm{~m}$. These are also in the experimental range. With these values given, we solve for $\Lambda_{2 T}$ and $F$ from (4.2) with (4.5). Then substituting into (4.4) and (3.3)-(3.5) with one of the strainenergy functions mentioned above, we can determine $\Lambda_{1 B}, \Lambda_{2 B}, \Lambda_{1 T J}$, $\Lambda_{2 J}$ and $\Lambda_{1 B J}$. Since these equations are highly nonlinear, we used NAG Library subroutine $c 05 N B F$ to find numerical solutions on a Pyramid 9810 superminicomputer.

Now we can evaluate $A=W-\lambda_{1}\left(\partial W / \partial \lambda_{1}\right)$ at $z \rightarrow \infty$ and $z \rightarrow-\infty$, and then determine $\lambda_{1}$ at an arbitrary point in terms of $\lambda_{2}$ from (2.7). For the strain-energy function with the form of (4.9), $\lambda_{1}$ can be expressed analytically in terms of $\lambda_{2}$. But for those with the form of (4.5) or (4.7), this is not the case and we used the Newton method in the determination of $\lambda_{1}$ in terms of $\lambda_{2}$. The tension resultants and the deformed configurations can be found from (2.4) and the first of (2.13) respectively. Another two quantities we are interested in are the original lengths $L$ of the parts of the membranes between the joining section and the section beyond which the angle $\omega$ is so small that the integration in (2.13) tends to be singular under the specified accuracy. These provide some information on the lengths of the artery and artificial graft for which the semi-infinite assumption can be used, and give a guide to the next section on the original length of the cuff.

Some of the numerical results obtained are shown in Tables 2-3. Table 2 follows from using (4.5) for the top part and (4.7) for the bottom part with the coefficients shown in Table 1 when $\Lambda_{1 T}=1.4$ at the top end section. The strain-energy function (4.9) for the bottom part and $\Lambda_{1 T}=1.4$ are used in Table 3. In the tables $L / \rho$ is the ratio of the original length, from the joining section to the section beyond which the angle $\omega$ is zero, to the original radius of the membrane.

From Tables 2-3, we can see that the jumps in $T_{2}$ at the joining section are larger in the orthotropic-isotropic cases than in the orthotropicorthotropic cases. So the How-Clarke orthotropic graft is better than the Mooney isotropic one from this point of view. To reduce the jump in $T_{2}$ around the joining section, we tried to change the material coefficients in (4.7) for the graft. From the calculations, we found that reduction in $D_{1}$ by 0.37 or $D_{4}$ by 0.43 makes the jump drop significantly when $\Lambda_{1 T}=1.4$ and $P_{0}=0.20 \times 10^{5} \mathrm{~N} / \mathrm{m}^{2}$, but reductions in $D_{3}$ by 1.2 or $D_{7}$ by 4.0 make the jump drop only slightly. This is mainly because $\lambda_{2}>\lambda_{1}$ at the joining 
TABLE 2. Values of extension ratios, axial force $F_{T}(N)$ at $z \rightarrow \infty$, resultant tensions $T_{1}$ and $T_{2}\left(\times 10^{2} \mathrm{~N} / \mathrm{m}\right)$ at joining and $L / \rho$ of joined Vaishnav and How-Clarke

semi-infinite membranes under pressure $P_{0}\left(\times 10^{5} \mathrm{~N} / \mathrm{m}^{2}\right)$ when $\Lambda_{1 T}=1.4$.

\begin{tabular}{|ccccccc|}
\hline$P_{0}$ & $\Lambda_{2 T}$ & $\Lambda_{1 T J}$ & $\Lambda_{2 J}$ & $\Lambda_{1 B J}$ & $\Lambda_{1 B}$ & $\Lambda_{2 B}$ \\
\hline 0.10 & 1.5093 & 1.6027 & 1.1658 & 1.0553 & 1.0762 & 1.0579 \\
0.15 & 1.7643 & 1.5829 & 1.3151 & 1.0621 & 1.0794 & 1.1081 \\
0.20 & 1.9727 & 1.5412 & 1.4780 & 1.0848 & 1.0632 & 1.1673 \\
\hline$P_{0}$ & $F_{T}$ & $T_{1 J}$ & $T_{2 T J}$ & $T_{2 B J}$ & $L_{T} / \rho$ & $L_{B} / \rho$ \\
\hline 0.10 & 0.8650 & 0.3870 & 0.2530 & 0.5750 & 4.0681 & 1.4237 \\
0.15 & 1.3140 & 0.4800 & 0.3310 & 1.0010 & 3.4890 & 2.2035 \\
0.20 & 1.7550 & 0.5360 & 0.4290 & 1.2510 & 3.7699 & 2.2102 \\
\hline
\end{tabular}

TABle 3. Values of extension ratios, axial force $F_{T}(N)$ at $z \rightarrow \infty$, resultant tensions $T_{1}$ and $T_{2}\left(\times 10^{2} \mathrm{~N} / \mathrm{m}\right)$ at joining and $L / \rho$ of joined Vaishnav and Mooney $\left(C_{1 B}=2.0 \times 10^{5} \mathrm{~N} / \mathrm{m}^{2}, C_{2 B}=0.4 \times 10^{5} \mathrm{~N} / \mathrm{m}^{2}\right)$ semi-infinite membranes under pressure $P_{0}\left(\times 10^{5} \mathrm{~N} / \mathrm{m}^{2}\right)$ when $\Lambda_{1 T}=1.4$.

\begin{tabular}{|ccccccc|}
\hline$P_{0}$ & $\Lambda_{2 T}$ & $\Lambda_{1 T J}$ & $\Lambda_{2 J}$ & $\Lambda_{1 B J}$ & $\Lambda_{1 B}$ & $\Lambda_{2 B}$ \\
\hline 0.10 & 1.5093 & 1.7067 & 1.0810 & 1.0084 & 1.0390 & 1.0092 \\
0.15 & 1.7643 & 1.7006 & 1.1821 & 0.9830 & 1.0374 & 1.0256 \\
0.20 & 1.9727 & 1.6644 & 1.2877 & 0.9571 & 1.0221 & 1.0496 \\
\hline$P_{0}$ & $F_{T}$ & $T_{1 J}$ & $T_{2 T J}$ & $T_{2 B J}$ & $L_{T} / \rho$ & $L_{B} / \rho$ \\
\hline 0.10 & 0.8650 & 0.3970 & 0.2810 & 0.7220 & 3.8362 & 1.1522 \\
0.15 & 1.3140 & 0.4970 & 0.3340 & 1.3490 & 3.4400 & 1.4417 \\
0.20 & 1.7550 & 0.5570 & 0.3740 & 1.9200 & 4.2333 & 1.3857 \\
\hline
\end{tabular}

section and $D_{1}$ and $D_{4}$ are directly involved in the expression for $T_{2}$ while $D_{3}$ and $D_{7}$ are involved only through the determination of $\lambda_{1}$ and $\lambda_{2}$.

The coefficients $D_{1}$ and $D_{4}$ are associated with the material property in the circumferential direction and $D_{3}$ and $D_{7}$ in the longitudinal direction. Reduction in $D_{1}$ and $D_{4}$ implies softening the material of the graft in the circumferential direction, and that in $D_{3}$ and $D_{7}$ implies softening the material in the longitudinal direction. From this point of view, it may be desirable to make the graft softer in the circumferential direction than the How-Clarke material. This softening procedure would result in a better match in circumferential compliance of the graft with the natural artery in which it is 
interposed. The effects of circumferential compliance on the failure at the joint were noted by Kidson and Abbott [5]. In an actual manufacturing process a material produced with say $D_{1}$ and $D_{4}$ changed in accordance with the indications given here may also have the other coefficients changed. Experiment and calculation would be then needed to determine the effect on the jump.

In Figures 2-4, we illustrate the deformed shapes and the variations in $T_{1}$ and $T_{2}$ when Vaishnav and How-Clarke semi-infinite membranes are joined together at the horizontal axis under the pressure $P_{0}\left(\times 10^{5} \mathrm{~N} / \mathrm{m}^{2}\right)=0.10$ (solid), 0.15 (dotted) and 0.20 (dashed) with $\Lambda_{1 T}=1.4$. We can see that the longitudinal resultant tension $T_{1}$ takes a maximum value at the joining and local minima in both the top and bottom parts when the pressure is relatively low $\left(P_{0}=0.10\right.$ and $\left.0.15 \times 10^{5} \mathrm{~N} / \mathrm{m}^{2}\right)$. When $P_{0}=0.20 \times 10^{5} \mathrm{~N} / \mathrm{m}^{2}, T_{1}$ has a local maximum at the joining and a local minimum in the top part. The jump in the circumferential resultant tension $T_{2}$ increases.as the pressure increases.

Since a membrane cannot withstand compression along its surface, the stress resultants $T_{1}$ and $T_{2}$ must be positive for any deformation. So is the strain-energy function. This places some restrictions on the coefficients

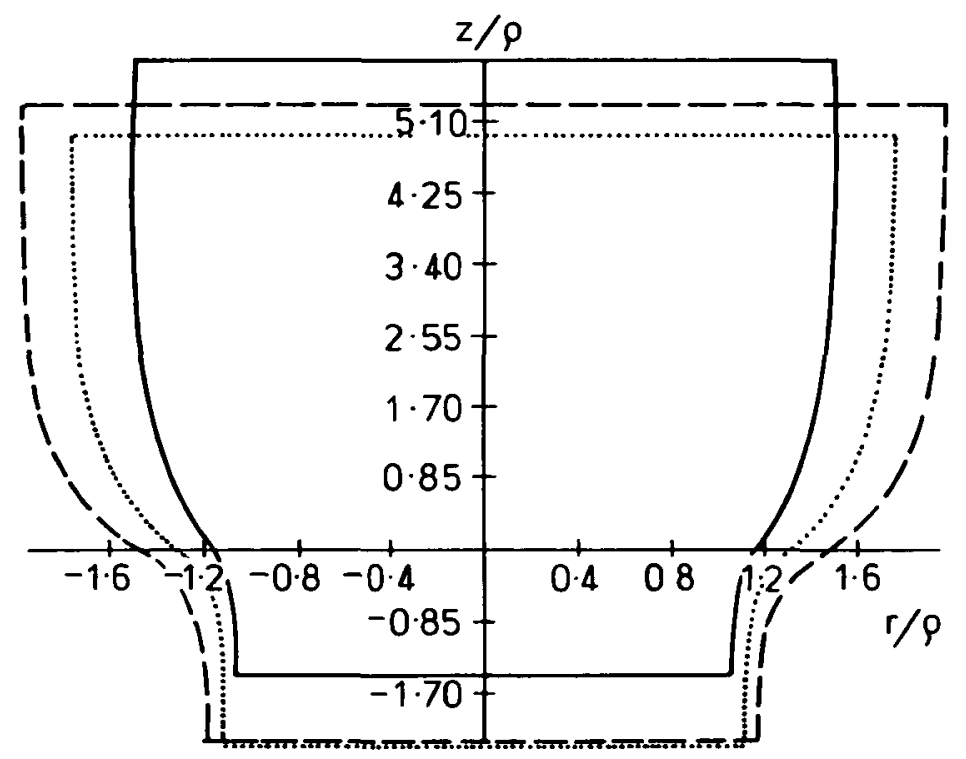

FIGURE 2. Deformed shape of joined Vaishnav (top) and How-Clarke (bottom) semi-infinite membranes under pressure $P_{0}\left(\times 10^{5} \mathrm{~N} / \mathrm{m}^{2}\right)=0.10$ (solid), 0.15 (dotted) and 0.20 (dashed) when $\Lambda_{1 T}=1.4$. The horizontal axis is the joining section in each case. 


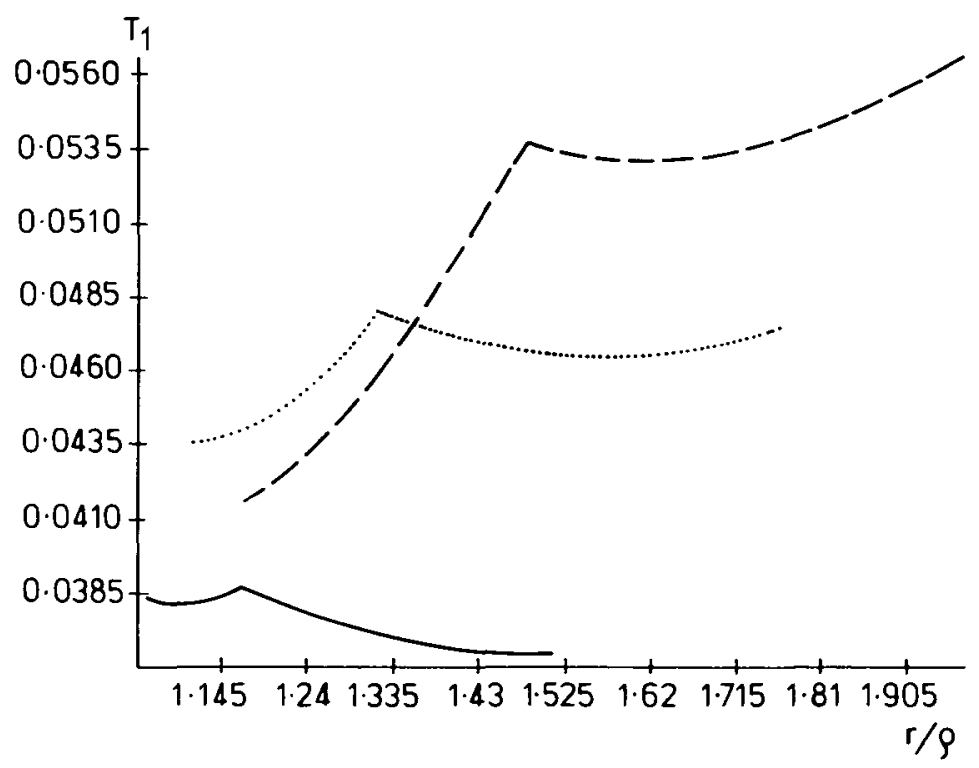

FiguRE 3. Variation of longitudinal resultant tension $T_{1}\left(\times 10^{3} \mathrm{~N} / \mathrm{m}\right)$ in joined Vaishnav (right) and How-Clarke (left) semi-infinite membranes under pressure $P_{0}\left(\times 10^{5} \mathrm{~N} / \mathrm{m}^{2}\right)=0.10$ (solid), 0.15 (dotted) and 0.20 (dashed) when $\Lambda_{1 T}=1.4$.

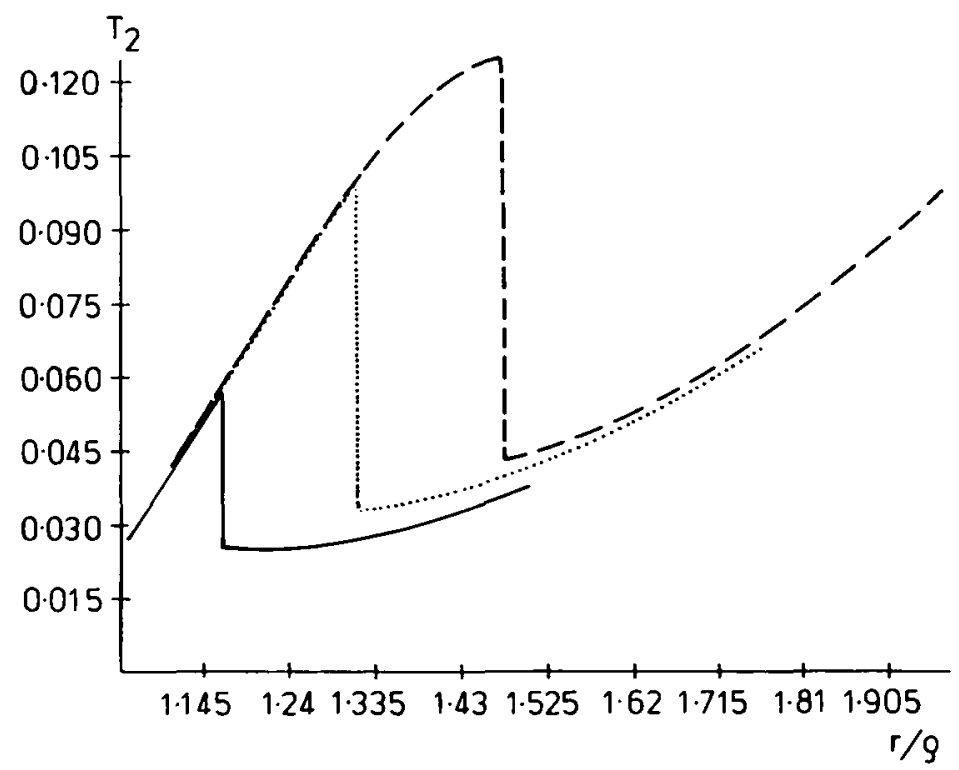

Figure 4. Variation of circumferential resultant tension $T_{2}\left(\times 10^{3} \mathrm{~N} / \mathrm{m}\right)$ in joined Vaishnav (right) and How-Clarke (left) semi-infinite membranes under pressure $P_{0}\left(\times 10^{5} \mathrm{~N} / \mathrm{m}^{2}\right)=0.10$ (solid), 0.15 (dotted) and 0.20 (dashed) when $\Lambda_{1 T}=1.4$. 
in the polynomial expressions in (4.5) and (4.7). By considering two special deformations in which $\lambda_{1}=1$ or $\lambda_{2}=1$, we find that the first three must be positive (this is a necessary condition, not sufficient), and the last four could be negative. If one, or more, of the last four are negative, then the deformations must be within some limits. From Table 1, we see that the first three coefficients are indeed positive in both (4.5) and (4.7), the seventh in (4.5) is negative but all of the last four in (4.7) are negative. So the deformations we consider here cannot exceed some limits. Instead of determining the limits, it is sufficient to check the signs for $T_{1}, T_{2}$ and $W$ during our calculations so that the results are valid. When we changed the coefficients $D_{i}$ in (4.7) to reduce the jump in $T_{2}$, we did the same.

\section{Two semi-infinite cylindrical membranes linked by a cuff}

The assumption in the previous section that the membranes are of infinite length is ideal because any membrane has a finite length. So we really need to deal with membranes with finite length. If we are interested in the implant of an artificial graft (cuff) with finite length $L_{C}$ into a natural artery we may model the natural arteries separated by the cuff as semi-infinite membranes. If we assume that the conditions at the infinite end sections of the natural arteries are the same and the internal pressure is constant, then the deformation is symmetric about the middle section of the cuff. Thus this problem can be treated as a join of a finitely long membrane (with half the length of the cuff) with a semi-infinite membrane. To simplify the following discussions, we refer to one of the semi-infinite membranes as the top part and the half cuff joining it as the bottom part. The extension ratios at the top end of the semi-infinite membrane and the joining are denoted as those in the previous section. The extension ratios at the symmetric section of the cuff are denoted as $\Lambda_{1 B}$ and $\Lambda_{2 B}$. Then the equations in (4.2) are still valid. Besides those equations in (3.3)-(3.5), we have two additional conditions from the bottom part: the one corresponding to (2.14) giving the original length of the half cuff and

$$
\Omega_{B}=0 \text { at } \lambda_{2 B}=\Lambda_{2 B} \text {, }
$$

the condition of symmetry at the middle section of the cuff. By making use of the equation corresponding to (2.12) for the bottom part and (3.6) with (4.3), this condition becomes

$$
\left.\left(\frac{\partial W_{B}}{\partial \lambda_{1 B}}\right)\right|_{\left(\Lambda_{1 B}, \Lambda_{2 B}\right)}=F+P\left(\Lambda_{2 B}^{2}-\Lambda_{2 T}^{2}\right) / 2 .
$$

Because of the symmetry condition (5.1), the integrals corresponding to 
(2.13) and (2.14) for the bottom part are singular at $\Lambda_{2 B}$. To avoid this singularity, we proceed as follows. Following Kydoniefs and Spencer [7] and expanding $\cos \omega_{B}$ in a series of $\left(\lambda_{2 B}-\Lambda_{2 B}\right)$ we find

$$
\cos \omega_{B}=1+g^{\prime}\left(\lambda_{2 B}-\Lambda_{2 B}\right)+O\left(\lambda_{2 B}-\Lambda_{2 B}\right)^{2},
$$

where

$$
g^{\prime}=\left.\left(d\left(\cos \omega_{B}\right) / d \lambda_{2 B}\right)\right|_{\lambda_{2 B}=\Lambda_{2 B}} .
$$

Then we have, in the neighbourhood of $\Lambda_{2 B}$

$$
\sin \omega_{B}=\left\{2 g^{\prime}\left(\Lambda_{2 B}-\lambda_{2 B}\right)\right\}^{1 / 2}+O\left(\Lambda_{2 B}-\lambda_{2 B}\right)^{\frac{3}{2}},
$$

and the condition giving the original length of the cuff may be written as

$$
L_{C} /(2 \rho)= \pm \int_{\Lambda_{2 B}}^{\Lambda_{2}} H\left(\lambda_{2 B}\right) d \lambda_{2 B} \mp\left[2 g^{\prime}\left(\Lambda_{2 B}-\Lambda_{2}\right)\right]^{\frac{1}{2}} /\left(\Lambda_{1 B} g^{\prime}\right),
$$

where

$$
H\left(\lambda_{2 B}\right)=\left(\lambda_{1 B} \sin \omega_{B}\right)^{-1}-\Lambda_{1 B}^{-1}\left[2 g^{\prime}\left(\Lambda_{2 B}-\lambda_{2 B}\right)\right]^{-\frac{1}{2}} .
$$

The integrand (5.7) takes the value zero at $\lambda_{2 B}=\Lambda_{2 B}$. The integrals corresponding to (2.13) for the bottom part can be written in a similar way.

Thus two kinds of problems can be considered:

(1) Given the radius after the deformation (equivalent to $\lambda_{2}=r / \rho$ ) of the cuff at the symmetric section, one is required to determine the original length of the cuff, the radius at the joining and the associated quantities such as the discontinuity in the circumferential tension at the joining section.

(2) Given the original length of the cuff, we are asked to determine the radii, which are equivalent to the circumferential extension ratios, of the cuff at the symmetry and at the joint; also the other quantities. In these problems, since $\left|\cos \omega_{B}\right| \leq 1, g^{\prime}\left(\lambda_{2 B}-\Lambda_{2 B}\right) \leq 0$. If $\lambda_{2 B}>\Lambda_{2 B}$ (or $\left.\lambda_{2 B}<\Lambda_{2 B}\right)$, which can be seen from the results for the semi-infinite membranes in the previous section, then only those values of $\Lambda_{1 B}$ and $\Lambda_{2 B}$ which produce negative (positive) $g^{\prime}$ can be used. To ensure $\left|\cos \omega_{B}\right| \leq 1$ near the symmetric section during the numerical calculation, we also need $\cos \Omega_{B}=1$. So we first find $\Lambda_{1 B}$ numerically from (5.2) for given $\Lambda_{2 B}$ or vice versa. Then (3.3)(3.5) and (5.6) serve as the governing equations for $\Lambda_{1 T J}, \Lambda_{2 J}, \Lambda_{1 B J}, \Lambda_{2 B}$.

For problems of the first kind $\Lambda_{2 B}$ is given, we determine $\Lambda_{1 B}$ numerically from (5.2) and then $\Lambda_{1 T J}, \Lambda_{2 J}, \Lambda_{1 B J}$ from (3.3)-(3.5). Finally the original length of the cuff is calculated from (5.6) and the other quantities follow. The problems of the second kind are more difficult than the first ones, since we need to solve the coupled highly nonlinear equations (3.3)-(3.5), (5.2) and (5.6) which involves an integration. The following numerical results are for these problems. 
The values of $L / \rho$ in the previous section provide some information about the original length of the cuff within the specified accuracy and whether or not it can be treated as a semi-infinite membrane. If $L_{C} /(2 p)$ is greater than $L_{B} / \rho$ of the previous section, then the cuff can be treated as a semi-infinite cylindrical membrane and the results of the previous section can be used. If $L_{C} /(2 \rho)$ is smaller than $L_{B} / \rho$, then the formulation outlined in this section can be employed. But the formulation in this section may still be used to carry out results when $L_{C} /(2 \rho)$ is slightly greater than $L_{B} / \rho$ of the previous section.

In the numerical calculations, we used the Vaishnav strain-energy function (4.5) for the top semi-infinite membrane, and the How-Clarke function (4.7), the Mooney function (4.9) as well as modified How-Clarke functions with different material coefficients for the cuff. The numerical results shown in Table 4 are obtained by using How-Clarke material for the cuff with the length $L_{C} /(2 \rho)=1.0$ while Table 5 is produced by using both How-Clarke (A) and modified How-Clarke ((B) with $D_{1}=2.6531,(\mathrm{C})$ with $D_{4}=-2.1259$, (D) with $D_{1}=2.8531$ and $\left.D_{4}=-1.8959\right)$ materials for the cuff with the length $L_{C} /(2 \rho)=2.0$ under the pressure $P_{0}=0.20\left(\times 10^{5} \mathrm{~N} / \mathrm{m}^{2}\right)$. In both Tables 4 and 5 , the longitudinal extension ratios at $z \rightarrow \infty$ of the top Vaishnav semi-infinite membrane is $\Lambda_{1 T}=1.4$.

In Figures 5-7, we illustrate the deformed shapes and the variations in $T_{1}$ and $T_{2}$ in the cases corresponding to the results shown in Table 5: (A) How-Clarke cuff-solid; (B) How-Clarke cuff with $D_{1}=2.6531$-dotted; (C) How-Clarke cuff with $D_{4}=-2.1259$-dashed; (D) How-Clarke cuff with $D_{1}=2.8531$ and $D_{4}=-1.8959$-dot-dashed. We can see that the How-Clarke cuff (A) produces a larger local maximum in $T_{1}$ at the joining section than (C)

TABLE 4. Values of extension ratios, axial force $F_{T}(N)$ at $z \rightarrow \infty$, resultant tensions $T_{1}$ and $T_{2}\left(\times 10^{2} \mathrm{~N} / \mathrm{m}\right)$ at joining and $L_{T} / \rho$ of joined Vaishnav semi-infinite membrane with How-Clarke cuff $\left(L_{C} /(2 \rho)=1.0\right)$ under pressure $P_{0}\left(\times 10^{5} \mathrm{~N} / \mathrm{m}^{2}\right)$ when $\Lambda_{1 T}=1.4$.

\begin{tabular}{|ccccccc|}
\hline$P_{0}$ & $\Lambda_{2 T}$ & $\Lambda_{1 T J}$ & $\Lambda_{2 J}$ & $\Lambda_{1 B J}$ & $\Lambda_{1 B}$ & $\Lambda_{2 B}$ \\
\hline 0.10 & 1.5093 & 1.6019 & 1.1666 & 1.0551 & 1.0725 & 1.0739 \\
0.15 & 1.7643 & 1.5807 & 1.3183 & 1.0617 & 1.0719 & 1.1495 \\
0.20 & 1.9727 & 1.5367 & 1.4870 & 1.0857 & 1.0532 & 1.2467 \\
\hline$P_{0}$ & $F_{T}$ & $T_{1 J}$ & $T_{2 T J}$ & $T_{2 B J}$ & $L_{T} / \rho$ & \\
\hline 0.10 & 0.8650 & 0.3870 & 0.2520 & 0.5770 & 4.0854 & \\
0.15 & 1.3140 & 0.4790 & 0.3320 & 1.0090 & 3.3272 & \\
0.20 & 1.7550 & 0.5350 & 0.4340 & 1.2550 & 3.7589 & \\
\hline
\end{tabular}


TABLE 5. Values of extension ratios, axial force $F_{T}(N)$ at $z \rightarrow \infty$, resultant tensions $T_{1}$ and $T_{2}\left(\times 10^{2} \mathrm{~N} / \mathrm{m}\right)$ at joining and $L_{T} / \rho$ of joined Vaishnav semi-infinite membrane with various cuffs $\left(L_{C} /(2 \rho)=2.0\right)$ : How-Clarke $(A)$ and modified How-Clarke $((B)$ with $D_{1}=2.6531,(\mathrm{C})$ with $D_{4}=-2.1259$, (D) with $D_{1}=2.8531$ and $D_{4}=-1.8959$ ) under pressure $P_{0}=0.20\left(\times 10^{5} \mathrm{~N} / \mathrm{m}^{2}\right)$ when $\Lambda_{1 T}=1.4$.

\begin{tabular}{|ccccccc|}
\hline$D_{i}$ & $\Lambda_{2 T}$ & $\Lambda_{1 T J}$ & $\Lambda_{2 J}$ & $\Lambda_{1 B J}$ & $\Lambda_{1 B}$ & $\Lambda_{2 B}$ \\
\hline$A$ & 1.9727 & 1.5412 & 1.4779 & 1.0848 & 1.0622 & 1.1744 \\
$B$ & 1.9727 & 1.4759 & 1.6361 & 1.1391 & 1.0543 & 1.2366 \\
$C$ & 1.9727 & 1.4891 & 1.5989 & 1.1155 & 1.0589 & 1.1989 \\
$D$ & 1.9727 & 1.4972 & 1.5777 & 1.1062 & 1.0577 & 1.2086 \\
\hline$D_{i}$ & $F_{T}$ & $T_{1 j}$ & $T_{2 T J}$ & $T_{2 B J}$ & $L_{T} / \rho$ & \\
\hline$A$ & 1.7550 & 0.5360 & 0.4290 & 1.2510 & 3.7691 & \\
$B$ & 1.7550 & 0.5310 & 0.5440 & 0.5590 & 3.5179 & \\
$C$ & 1.7550 & 0.5310 & 0.5110 & 0.5750 & 3.5844 & \\
$D$ & 1.7550 & 0.5310 & 0.4950 & 0.0767 & 3.6266 & \\
\hline
\end{tabular}

TABLE 6. Jumps in $T_{2}\left(\times 10^{2} \mathrm{~N} / \mathrm{m}\right)$ and $\lambda_{1}$ at the joining under the various pressures $P_{0}\left(\times 10^{5} \mathrm{~N} / \mathrm{m}^{2}\right)$ and $\Lambda_{1 T}=1.4$ when Vaishnav and How-Clarke (A) or modified How-Clarke ((B) with $D_{1}=2.6531$, (C) with $D_{4}=-2.1259$, (D) with $D_{1}=2.8531$ and $D_{4}=-1.8959$ ) semi-infinite membranes are joined together.

\begin{tabular}{|ccccc|cccc|}
\hline \multicolumn{5}{|c|}{$T_{2}$} & & \multicolumn{3}{c|}{$\lambda_{1}$} \\
\hline$P_{0}$ & 0.10 & 0.15 & 0.19 & 0.20 & 0.10 & 0.15 & 0.19 & 0.20 \\
\hline$A$ & 0.3220 & 0.6700 & 0.8240 & 0.8220 & 0.5474 & 0.5208 & 0.4736 & 0.4564 \\
$B$ & 0.2930 & 0.5540 & 0.4540 & 0.0950 & 0.5342 & 0.4999 & 0.4244 & 0.3517 \\
$C$ & 0.3090 & 0.5850 & 0.4760 & 0.0950 & 0.5448 & 0.5116 & 0.4411 & 0.3782 \\
$D$ & 0.3030 & 0.5780 & 0.5060 & 0.2780 & 0.5403 & 0.5072 & 0.4379 & 0.3920 \\
\hline
\end{tabular}

and (D), and (B) does not produce a local maximum at all. It can also be seen that the jumps in $T_{2}$ produced by the modified How-Clarke cuffs, especially by (B), are very small. It is also noted that the modified How-Clarke cuffs have larger radii so that the fluid (blood) can pass through more easily than through the How-Clarke one. The symmetric sections of the cuffs are located at the horizontal axis and the joining sections are at $z=z_{0}$. It can be seen that the joining sections are very close to each other. 


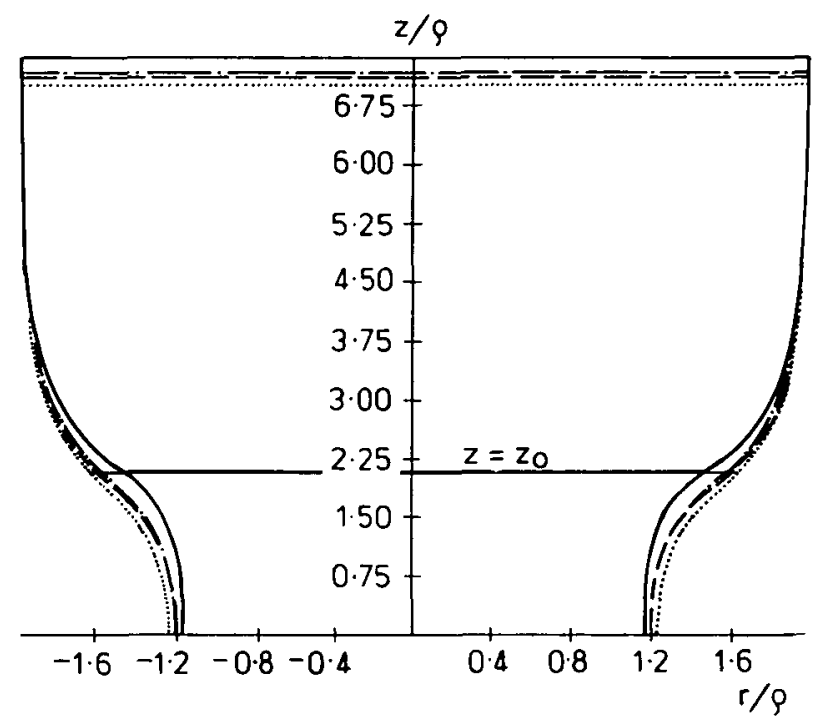

Figure 5. Deformed shape of joined Vaishnav semi-infinite membrane $\left(z \geq z_{0}\right)$ with various cuffs $\left(0 \leq z \leq z_{0}\right.$ with $\left.L_{C} /(2 \rho)=2.0\right)$ : How-Clarke (A)-solid and modified How-Clarke ((B) with $D_{1}=2.6531$-dotted, (C) with $D_{4}=-2.1259$-dashed, (D) with $D_{1}=2.8531$ and $D_{4}=-1.8959$-dot-dashed) under pressure $P_{0}=0.20\left(\times 10^{5} \mathrm{~N} / \mathrm{m}^{2}\right)$ when $\Lambda_{1 T}=1.4$. The horizontal axis is the symmetric sections of the cuffs. The joining sections are at $z=z_{0}$, very close in each case.

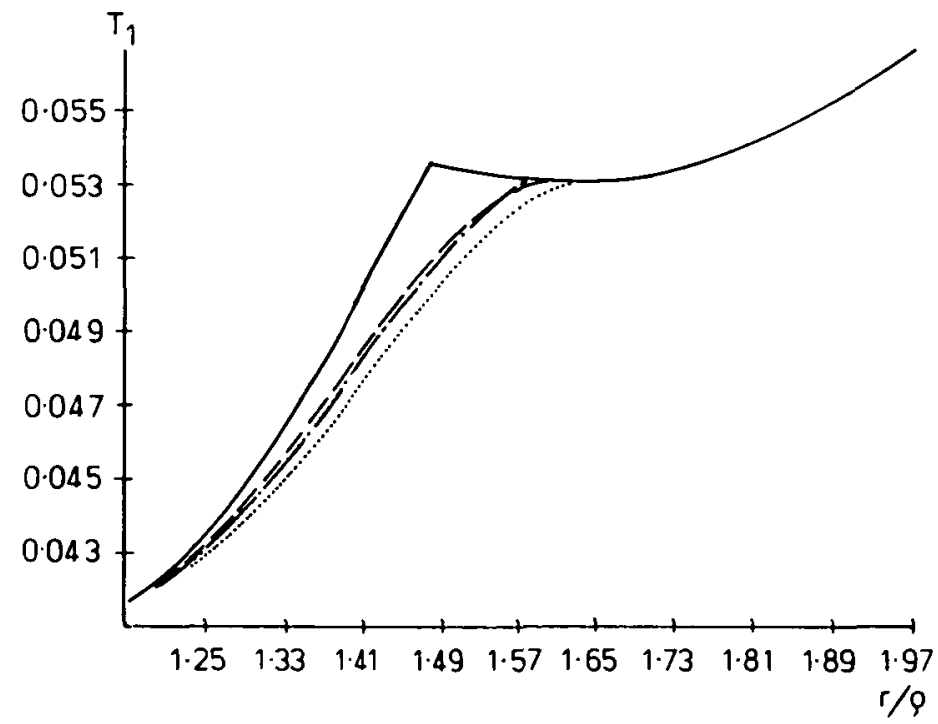

FIGURE 6. Variation of longitudinal resultant tension $T_{1}\left(\times 10^{3} \mathrm{~N} / \mathrm{m}\right)$ in joined Vaishnav semi-infinite membrane (right) with various cuffs (left with $L_{C} /(2 \rho)=2.0$ ): How-Clarke (A)solid and modified How-Clarke ((B) with $D_{1}=2.6531$-dotted, (C) with $D_{4}=-2.1259$-dashed, (D) with $D_{1}=2.8531$ and $D_{4}=-1.8959$-dot-dashed) under pressure $P_{0}=0.20\left(\times 10^{5} \mathrm{~N} / \mathrm{m}^{2}\right)$ when $\Lambda_{1 T}=1.4$. 


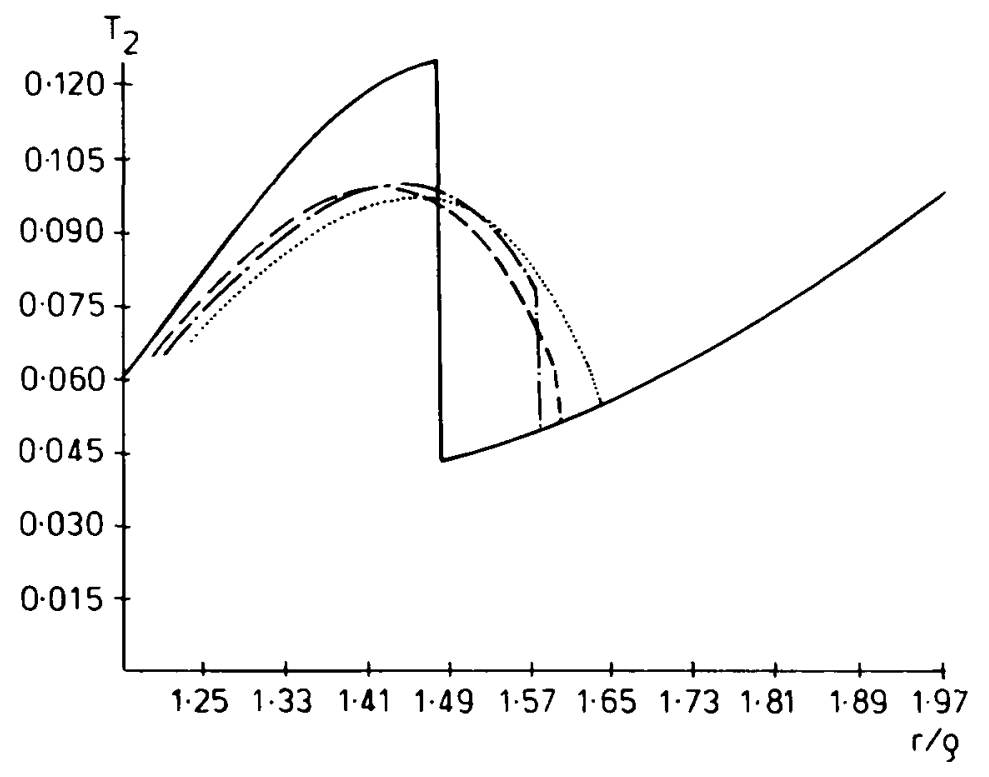

FIGURE 7. Variation of circumferential resultant tension $T_{2}\left(\times 10^{3} \mathrm{~N} / \mathrm{m}\right)$ in joined Vaishnav semi-infinite membrane (right) with various cuffs (left with $L_{C} /(2 \rho=2.0)$ : How-Clarke (A)solid and modified How-Clarke ((B) with $D_{1}=2.6531$-dotted, (C) with $D_{4}=-2.1259$-dashed, (D) with $D_{1}=2.8531$ and $D_{4}=-1.8959$-dot-dashed) under pressure $P_{0}=0.20\left(\times 10^{5} \mathrm{~N} / \mathrm{m}^{2}\right)$ when $\Lambda_{1 T}=1.4$.

\section{Discussion}

When we consider the results for two semi-infinite membranes joined together we note, as shown in Table 6, that for a given material, as the pressure increases, the jump in the longitudinal extension ratio $\lambda_{1}$ decreases while that in the circumferential tension $T_{2}$ first increases then decreases. For $T_{2}$ in a How-Clarke graft the biggest jump takes place when $P_{0}=0.19 \times 10^{5} \mathrm{~N} / \mathrm{m}^{2}$, but in the modified How-Clarke grafts the maximum jump occurs at $P_{0}=$ $0.15 \times 10^{5} \mathrm{~N} / \mathrm{m}^{2}$. But for any pressure in the range considered the modified How-Clarke grafts have smaller jumps in both $T_{2}$ and $\lambda_{1}$ than the HowClarke one. Therefore from this point of view, the modified How-Clarke grafts are better than the How-Clarke one. These results are almost identical with those found for the cuff joined to the semi-infinite membrane as illustrated in Figures 5 to $7 P_{0}=0.20 \times 10^{5} \mathrm{~N} / \mathrm{m}^{2}$.

Extensive numerical results were obtained for the deformation of the mem- 
branes with and without a cuff interposed, and some of them are given in tabular and graphical form. In particular these results display the discontinuity in the circumferential stress at the joint, and, as described above, we note that this discontinuity can be significantly reduced by varying certain elastic coefficients in an expression for the orthotropic strain-energy function used by How and Clarke [4]. This may have some implication for the design of artificial implants since leaking at the joint is an outstanding problem. But the influence of dynamic rather than static pressure would need to be addressed in this context. It is not claimed that the solutions found here are unique, but they are consistent with observation of cylinders deformed under the relevant conditions.

Since by (2.2) the half deformed thickness $h=h_{0}\left(\lambda_{1} \lambda_{2}\right)^{-1}$, it is also discontinuous at the joining section. The relative discontinuity in $h / h_{0}$, which is $\pm\left(\Lambda_{1 T J}-\Lambda_{1 B J}\right)\left(\Lambda_{2 J} \Lambda_{1 T J} \Lambda_{1 B J}\right)^{-1}$, is generally smaller than the relative change in $T_{2}$, with exception of the cases of modified How-Clarke materials. The modified How-Clarke materials also produce smaller discontinuities in the deformed thickness than the other materials. The effects of the discontinuity in the deformed thickness may need to be considered in more detail when one studies the flow of blood in the vessels.

\section{Acknowledgement}

One of the authors, J. Shi, is very grateful to the University of Queensland for financial support.

\section{References}

[1] J. E. Adkins and R. S. Rivlin, "Large elastic deformations of isotropic material, IX: the deformation of thin shells", Phil. Trans. Roy. Soc. London A 244 (1952) 505-531.

[2] D. H. Bergel, "The properties of blood vessels", in Biomechanics: Its Foundations and Objectives, (eds. Y. C. Fung, N. Perrone \& M. Anliker) (Prentice-Hall, Englewood Cliffs, New Jersey, 1972) Chapter 5.

[3] V. G. Hart and Jingyu Shi, "Joined dissimilar isotropic elastic cylindrical membranes under internal pressure and longitudinal tension", Quart. J. Mech. Appl. Math. 44 (1991) $581-600$.

[4] T. V. How and R. M. Clarke, "The elastic properties of a polyurethane arterial prothesis", J. Biomechanics 17 (1984) 597-608.

[5] I. G. Kidson and W. M. Abbot, "Low compliance and arterial graft occlusion", Cardiovasc. Surg. 58 (1978) I-1-I-3.

[6] A. D. Kydoniefs, "Finite axisymmetric deformations of an initially cylindrical elastic membrane enclosing a rigid body", Q. J. Mech. Appl. Math. 22 (1969) 319-331.

[7] A. D. Kydoniefs and A. J. M. Spencer, "Finite axisymmetric deformations of an initially 
cylindrical elastic membrane”, Quart. J. Mech. Appl. Math. 22 (1969) 87-95.

[8] T. J. Pedley, The fluid mechanics of large blood vessels, (Cambridge University Press, New York, 1980).

[9] A. C. Pipkin, "Integration of an equation in membrane theory", ZAMP 19 (1968) 818819.

[10] R. S. Rivlin and D. W. Saunders, "Large elastic deformations of isotropic materials. VII: Experiments on the deformation of rubber", Phil. Trans. Roy. Soc. London A 243 (1950-51) 251-288.

[11] R. N. Vaishnav, "Mathematical characterization of the nonlinear rheological behavior of the vascular tissue", Biorheology 17 (1980) 219-226. 